\title{
Author Index, Abstracts
}

Numbers refer to Abstract No.

\author{
Agerlin, N. 1,110 \\ Aguiar, P.H. 47 \\ Aguiar, S. 64 \\ Aguzzi, A. 8 \\ Aimard, L. 124 \\ Alainard, J.L. 113 \\ Alapetite, C. 57, 113,124 \\ Albright, A.L. 55,85,115, 118 \\ Allegranza, A. 30 \\ Allen, J. 59,60,65 \\ Allen, J.C. 55,85, 118 \\ Alterman, R.L. 24 \\ Amsallem, D. 61 \\ Andreussi, L. 17 \\ Aricò, M. 16 \\ Artaryan, A.A. 19 \\ Ater, J.L. 81 \\ Bábel, B. 116 \\ Babin-Boilletot, A. 61 \\ Balestrini, M.R. 92 \\ Ballerini, E. 73 \\ Barbara, A. 71 \\ Barszcz, S. 74 \\ Barthez-Carpentier, M.A. 51 \\ Basso, G. 66 \\ Batra, S.K. 39 \\ Battistella, P.A. 66 \\ Bauer, J. 88 \\ Bayle Bustamante, G. 101 \\ Beck, J.D. 72, 94 \\ Becske, T. 116 \\ Behnke, J. 20 \\ Belin, M.F. 18, 117 \\ Bellantoni, G. 16 \\ Beneš, V. 88 \\ Benhassel, M. 89, 113 \\ Benk, V. 49 \\ Bennett, D. 100
}

Berger, M.S. 13,25,26,34,65,

106, 115 Bergeron, C. 89 Bergesen, S.E. 1 Berghorn, W.J. 114 Bernard, J.-L. 79,80,124 Bernheim, A. 40 Bersnev, V.P. 119 Besenzon, L. 17,71,75 Biegel, J. 33 Bigner, D.D. 39 Bigner, S.H. 39 Billaniuk, L.T. 42 , 86 Bleyer, W.A. 55,85,118 Bobola, M.S. 13 Bodey, B. 21 
Bognar, L. 83

Børgesen, S.E. 110

Borsi, J. 116

Bosch, D.A. Ill

Bothwell, M. 26

Bouffet, E. 57, 58, 79, 80, 83,

117, 124 Bouknik, H. 49 Boyett, J. 55,56,85,118 Brach del Prever, A. 71, 75,

103 Bradley, M.K. 24 Brand, W.N. 122 Brem, H. 14, 15 Brock, M. 10 Brown, M. 76 Bruce, D.A. 56,87 Brugières, L. 78 Brunat-Mentigny, M. 57, 79 ,

80, 124 Bsili, L. 67 Buffo, A. 27 Buhl, Ll 110 Burger, P.C. 54 Busson, A. 113

Cáceres, A. 22 Calvo-Escribano, C. 22 Calvo-Manuel, F. 97 Cama, A. 103 Carboné, A. 22 Carli, M. 66 Carrie, C. 57, 113, 124 Caudry, M. 108 Cavalla, P. 27 Cefalo, G. 73 Celerier, D. 108 Cerejo, A. 35 Chadduck, W. 56 Chastagner, P. 61 Chatterjee, S. 45 Chauvin, F. 58 Chazal, J. 44 Cherlow, J. 55 Chess, M. 90 Chiabotto 103 Chiò, A. 27 Chodounský, Z. 88 Choux, M. $38,58,80,124$ Ciquini, O. 47 Cogen, P.H. 36 Cohen, B. 56 Cohen, M.E. 54 Colosimo, C. 77 Colvin, O.M. 14, 15 
Cordero di Montezemolo, L.

71,75 Corrias, A. 17, 103 Cuccia, V. 12,121 Cumlivská, E. 88 Czaykowski, D. 52

Daneshvar, L. 36 D’Angio, G.J. 84 Danjoux, C. 2 Darcel, F. 89 Das, Lل 122 Deb, G. 105 Delumley, L. 61 Demaille, A. 57 Demaille, M.C. 113 Demeaux, H. 108 Dengg, K. 93 DeSanctis, C. 17 Deutsch, M. 55 Devoti, M. 99 Diaz, D. 12

Diez, B. 46,98, 101,104 DiRocco, C, 77,105 Di Telia, M. 12 Dobrovsky, W. 93 Donfrancesco, M. 105 Dörr, H. 72,94 Doz, F. 57,79,80, 124 Dramińská, D. 74 Dufay, N. 18, 117 Duffner, P.K. 54 Duhaime, A.-C. 7 Durand, C. 4 Dweik, A. 51

Edan, C, 61,89 Edwards, M.S.B. 36,63 Elterman, R.D. 56,87 Epelman, S. 64 Epstein, F.J. 59 Erhardt, J. 72,94 Erichsen, G. 53 Etchepareborda, M. 98 Evren Keles, G. $25,26,106$ Ewers,|T.G. 13 Eys, J. van 81 Fahlbusch, R. 72 Fazekas, I. 116 Ferreira, G. 35 Ferrell, L. 76 Ferrigo, R. 64 Fiedler, A. 123 Figarella-Branger, D. 23 
Fink, F.M. 93

Finlay, J.L. 55,59,60,65,85

118 Firth, I. 95 Foley, B. 100 Fontan, D. 108 Fossati-Bellani, F. 73, 99 Frappaz, D. 80 Freeman, C.R. 54 Freycon, F. 124 Friedman, H.S. 39 , 54,76 Fritsch, E. 93 Frydrych, A. 123 Fuchs, H. 76

Gallego, P. 22 Gallo, G. 12

Gambarelli, D. 23, 38, 80 Gamboni, M. 28 Gandola, L. 73 Garcia-Pérez, A. 97 Gardas-Skowrońska, A. 74 Garré, L. 103 Garvin, J. 59 Gasparini, M. 73 Gautris, P. 107,108 Gejeman, G. 24 Gene, R. 101 Genitori, L. 38 Gennarelli, L. 43 Gentet, J.-C. 23,38,57,58,79,

80, 124 Gerard, J.P. 113 Geyer, J. 85 Geyer, R. 55,65,118 Gil-da-Costa, M.J. 35 Gimenez-Más, J.A. 22 Giombini, S. 92 Giordana, M. T. 27 Giordano, L. 92 Giorgi, Cl 99 Giraudon, P. 18 Gjerris, F. 1,53,110

Goedrich, J.T. 24 Goldstein, G.W. 9 Goldwein, J. 52,55,56,81,86,

96, 100 Gonçalves, M. 35 Gouyette, A. 29,40 Grabenbauer, G. 72,94 Grajkowska, W. 74 Greenberg, M. 2 Grovas, A. 60 Gruccio, D. 102 Guarnieri, J. 67

336

Guegan, Y. 89 Guerin, Cl 9 Guérin, J. 107,108 Guyotat, J. 83

Haase, J. 110

Habrand, J.L. 49,78

Haie, C. 113

Halpern, S. 60

Hans, F.J. 82

Harkness, W. 109

Hartmann, O. 78

Hayward, R. 109

Hertz, H. 53

Hirsch, J.-F. 29,31,37,40,58,

91 Hockenberger, B. 76 Hockley, A. 70 Hoffman, HJ. 2 Hoffstetter, S. 113 Honorato, D.C. 64 Hoppe Hirsch, E. 91 Horowitz, M.E. 54 Houtteville, J.P. 67 Hovind, K, H. 69 Huber, W. 93

Iannelli, A. 105 Ipola, E. de 98,101 Israel, M. 55

Jacobi, G. 41,123 Jakacki, R. 59, 60, 65, 85 James, H.E. 54 Jan, M. 51 Jansen, H.-Th. 3 Janss, A.J. 120 Jaspan, T. 48 Jenkin, D. 2 Jennings, M. 65 Jimenez, D.F. 24 Joannard, A. 4 Johnston, H. 62 Jones, R. 62 Jouvet, A. 80,83,117

Kalifa, C. 29,31,37,40,49,58,

61,78,79 Kálmánchey, R. 116 Kao, G.D. 96 Kaufmann, S.H. 14 Kawamata, M. 32 Kazak, A. 100 Kchuchlaeva, E. 50 Kellie, S. 62 Kerby, T. 76 Kerr, C. 113 Khachatryan, V.A. 119 Kirschner, J.P. 54 Klinken, L. 110 
Kochi, M. 68 Kolodié, H| 4, 113 Komarov, V. 50 Korschunov, A. 50 Kraker, J.de 111 Kubo, O. 32 Kun, L.E. 54 Kuratsu, J. 68 Kurcznski, E. 65 Kurisaka, M. 112

Lachance, D. 76 Lacombe, M.J. 31 Lagrange, J.L. 113 Lala, R. 17 Lamagnère, J.P. 51 Lange, B. 52, 56, 65, 86, 96,

100, 120 Lange, J.C. 67 Lapras, C, 80,83,124 Lasio, G. 30, 92 Lasorella, A. 77 Lasset, C. 58, 113 Laterra, J. 9 Lauffer, H. 72 Laverda, A.M. 66 Lee, V.Y. 120 LeGall, E. 89 Leggate, J. 48 Lejars, O. 61 LellouchTubiana, A. 29,31 ,

37,40 Lemaire, J.J. 44 Lemerle, J. 37, 78 Leveratto, L. 17 Levin, V.A. 63 Levy, N. 40 Lim, R. 106 Lima Reis, M.I. 35 Lindsley, K. 59 Locatelli, D. 16,75 Lombardi, F. 73 Ludwig, H.-Ch. 20 Lundar, T. 69

McDonald, J.D. 36 McDowell,H. 45 McElwain, M. 60 McGuire, P. 85,118 Mclntosh, S. 60 Mack, E. 36 McLaughlin, J. 2 McLendon, R.E. 39 McLone, D.G, 122 McWhirter, W. 62 Madon, E. 71,75 Magrassi, L 16, 75 Maheut-Lourmière, J. 51 Maire, H.Ph. 108 Make, J.P. 107, 113 
Malcus, C. 18 Mališ, J. 88 Malocco, F. 66 Mann, J. 70 Marino, R., Jr. 47 Marino, S. 27 Markakis, E. 20 Martinez, M. 28 Martínez-Lanao, D. 22 Marymont, M.H. 122 Mastrangelo, R. 77 , 105 Mastrangelo, S. 77 May, P.L. 45 Mazewski, C. 65 Mazingue, F. 79 Mazzi, J. 101 Mechinaud, F. 78 Meier,W. 94 Melaragno, R. 64 Merkt, Ch. 41 Metzger, A.K. 36 Meyers, S.P. 90 Micheli, R. 92 Michon, J. 57 Mihara, Y. 68

Milani, N. 99 Milstein, J. 85, 118 Mircevski, V. 83 Moghrabi, A. 76 Molenaar, W.M. 120 Mollica, F. 16 Molloy, P. 52 Monges, J. 12, 104, 121 Mori, K. 112 Mortensen, A.D. 110 Moshang, T., Jr. 102 Mottl, H. 52 Mottolese, C. 58,83,117 Mulhern, R.K. 54 Mulney, L. 56 Munzer, M. 61 Muracciole, X. 113 Muramoto, M. 13

Narbena-García, J. 97 Needle, M. 81 Negrier, S. 113 N’Guyen, T.D. 113 Nicholson, H.S. 55, 56 Niedorf, H.R. 3

Oberbauer, R. 93 Oette, D. 76 O’Reilly, G. 109 Ostertag, C.B. 82 Ovesen, N. 110 Ozerova, V. 50 
Pacheco, G. 28

Packer, R.J. 7, 55, 56, 65, 84,

85,118 Pakisch, B. 93 Pantaleoni, C. 99 Paraicz, E. 116 Parkes, S. 70 Parrish, J.M. 100 Pasquier, B. 4 Passagia, J.-G. 4 Pastan, I. 15 Pedersen, H. 53 Pellissier, J.F. 23 Pellone, M. 66 Perilongo, G. 66, 120 Pezzotta, S. 16,71,75 Philip, T. 58 Phillips, P.C. 14,15,42,52,56,

$84,86,100,102,120$ Picco, P. 17,46,103,104 Pillwein, K. 93 Pinto, |N. 113

Plantaz, D. 4,61,79,80,124 Plese, J.P.P. 47 Polio, B. 30 Pomata, H. 12, 121 Pons, A. 113 Ponvert, D. 57 Portillo, S. 46 Pospiech, J. 3 Povýšilová, A. 88 Prados, M.D. 55,63 Pröschel, U. 94 Puccetti, D. 60 Punt, J.

Radcliffe, J. 96, 100 Raevskai, S. 50 Raquin, M.A. 49,61 Raslawsky, E. 121 Raybaud, C. 38, 57, 79, 80 Reaman, G. 56 Reboul, A. 18 Rebsamen, S. 86 Reinhardt, V. 3 Remón, L. 22 Reske-Nielsen, E. 110 Riccardi, R. 77, 105 Rigobello 66 Rilliet, B. 5 Riou, G. 37 Riva, D. 99 Rivel, J. 107,108 Robson, K. 48 Roche, H. 57,79,80,124 Rorke, LB. 6, 7, 43, 84, 85,

118, 120 Rosenblum, M. 59 Rossi, M. 101 Roszkowski, M. 74

Rottoli, L. 73 Rougon, G. 23 Roux, N. 117 Rozan, R. 113 Russell Geyer, J. 115

Sadhev, I. 60 Sainte-Rose, C. 31 Salvagiloi, JV. 64 Sanematsu, P. 64 Sanford, R.A. 54 SanGalli, F. 107, 108 Santini, J J. 51 Sariban, E. 79 Sarrazin, D. 49 Sauer, R. 72 Savoiardo, M. 73 Schiff, S. 56 Schiffer, D. 27 Schilter, B. 5 Schmidt, K. 110 Schofield, D. 26, 115 Schold, C. 76 Schuler, D. 116 Schultz, M. 12,28,104 Schut, L. 7 , 56 Schwabe, D. 123 Scorzo, G. 101 Seidel, F.G 54 Seng, S.H. 113,124 Seto, H. 68 Sevlever, G. 12,28,46, 104

121 Seyer, H. 72,94 Shimoda, M. 32 Shurin, S. 85,118 Sierrasesumaga, L. 97 
Silber, J.H. 120 Silber, J.R. 13,25,34, 106 Slave, I. 93 Slovik, F. 116 Smith, G. 70 Sokal, M. 48 Solero, C.L. 30, 73, 92 Somló, P. 116 Sosa, P. 121 Sotti, G. 66 Spooner, D. 70 Spreer, J. 41 Srinivasin, J. 34 Stanley, P. 85,118 Stehbens, J. 85, 118 Stela, C.R. 64 Stevens, G. 95 Stevens, K. 85,118 Stevens, M. 70 Stewart, B. 76 Strauss, L.C. 122 Sure, U. 114 Sutton, L.N. 7, 42, 43, 56, 84, 86,96,100,120

Tajika, Y. 32

Takaki, S. 68

Takakura, K. 32

Tan, W.F. Ill

Taratuto, A.L. $12,28,46,104$

121 Taylor, C.R. 21 Teper, A. 101

Terrier-Lacombe, M.-J. 29, 40 Tesoro-Tess, J.D. 73 Thyss, A. 57,80,124 Tien, R. 76 
Tohyama, T. 32, 120 Tomita, T. 122 Toogood, I. 62 Topczewiski, A. 64 Tornesello, A. 77 Torres, C. 86 Tóth, K. 116 Trojanowski, J.Q. 11,120 Tron, P. 57,79,80, 124 Tugal, O. 60 Turkowski, Z. 74 Turmo, M. 107 Twerdy, K. 93

Uemura, S. 68 Urban, |Ch. 93 Urgesi, A. 71 Ushio, Y. 68

Vagner-Capodano, A.M. 38 Valente, A. 77 Valentini, L.G. 30 Valery, C.A. 29, 37 Valteau, D. 78 Vassal, G. 29,31,37,40,78 Vaz, R. 35 Vekemans, M. 31 Veling, G. 82 Venuat, A.-M. $29,31,40$ Vigliani, M.C. 27 Villablanca, J. 65 Vital, A. 107 Vital, C. 107 Vloeberghs, M. 48

Wacker, P. 5 Wagner, J.P. 113 
Walker, D. 48 Walker, R.W. 59,60 Walsh, R. 70 Wang, Z. 43 Wara, W.M. 63 Warnke, P.C. 82 Waters, K. 62 Weber, B. 90 Weber, F. 10 Wehrli, S. 43 Wenzel, D. 94 White, L. 62 Wild, K. von 3 Wildenhain, S. 90 Willert, J.R. 36 Willoughby, M. 65 Wisoff, J. 59,85,118 Wocjan, J. 74 Wolff, J.E.A. 9 Wyss, M. 5

Yachnis, A.T. 120 Yakovenko, I.V. 119 Yssing, M. 53 Yung,|K.|A. 81

Zamaróczy, D. 116 Zattara-Cannoni, H. 38 Zeltzer, P. 55, 85, 118 Zhu, J. 10 Zimmerer, B. 20 Zimmerman, R.A. 42, 43 Zorzi, C, 99 Zucker, J.M. 57,79,80,124 
